\title{
Characterization of an annular four-channel Silicon Drift Detector with a light element window
}

\author{
R. Terborg, M. Rohde \\ Bruker AXS Microanalysis GmbH, Schwarzschildstr. 12, 12489 Berlin, Germany
}

Since its introduction of energy dispersive x-ray detection, the Silicon Drift Detector (SDD) has become increasingly popular and is the process of replacing the lithium-drifted silicon detector $\mathrm{Si}(\mathrm{Li})$ for good.

State of the art SDDs are able to outperform $\mathrm{Si}(\mathrm{Li}) \mathrm{s}$ in almost every aspect. High resolution type SDDs have an energy resolution of $123 \mathrm{eV}$ or better at $\mathrm{Mn}-\mathrm{K} \alpha$. Optimized detector radiation entrance windows [1] minimize shelf and tail effects down to energies of $108 \mathrm{eV}$ (Beryllium-K $\alpha$ ) and show undistorted Gaussian peak shapes with an energy resolution of $\leq 54 \mathrm{eV}$ at F-K $\alpha$ and $\leq$ $46 \mathrm{eV}$ at $\mathrm{C}-\mathrm{K} \alpha$. Optimized electronics maintain this energy resolution even for count rates up to 100 kcps input count rate.

Multiple element SDD configurations with four separate detectors integrated onto one chip but with separate electronics, provide even higher count rates through increased active area without increasing pile-up or dead time [2,3]. The multi element concept also allows a larger solid angle without degrading energy resolution.

Fig. 1 shows a four element structure consisting of four $15 \mathrm{~mm}^{2}$ SDDs, leading to an effective detector area of $60 \mathrm{~mm}^{2}$. Fig. 2 shows the implementation of this detector chip into a specially designed detector, the QUAD XFlash 5060, which has an optimized geometry in order to be placed in a setup between the pole piece and the sample within a standard SEM like a BSE detector, fig. 3. The detector has a hole in the center for the primary beam. Therefore the four SDDs are centered around the scanning electron beam path, covering a solid angle of about 0.9 sr which makes it an ideal device for high speed mapping applications, fig. 4. The best energy resolution is about $127 \mathrm{eV}$ at $\mathrm{Mn}-\mathrm{K} \alpha$ and the maximum output count rate is about $1,000,000 \mathrm{cps}$.

The detector can be equipped with a polymer window in order to prevent backscattered electrons from entering the detector. Different types of windows can be used depending on the low energy efficiency and the energy of the backscattered electrons.

The aim of this presentation is to show a characterization of a flat QUAD annular detector, the count rate performance, light element sensitivity and mapping properties.

References

[1] A. Niculae et al., Microsc. Microanal. 13 (Suppl.2) (2007), 1430

[2] R. Terborg, M. Rohde, Microsc. Microanal. 14 (Suppl.2) (2008), 1166

[3] P. Kotula et al., Microsc. Microanal. 14 (Suppl.2) (2008), 116 


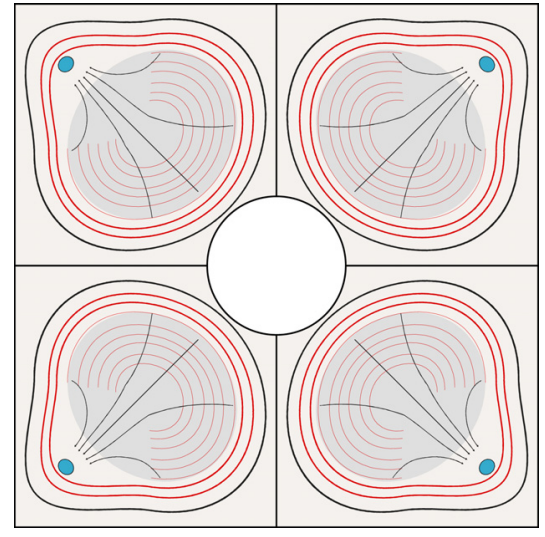

Fig. 1. Design of a four channel detector structure consisting of four $15 \mathrm{~mm} 2$ SDDs which has a hole in the center for the primary beam.

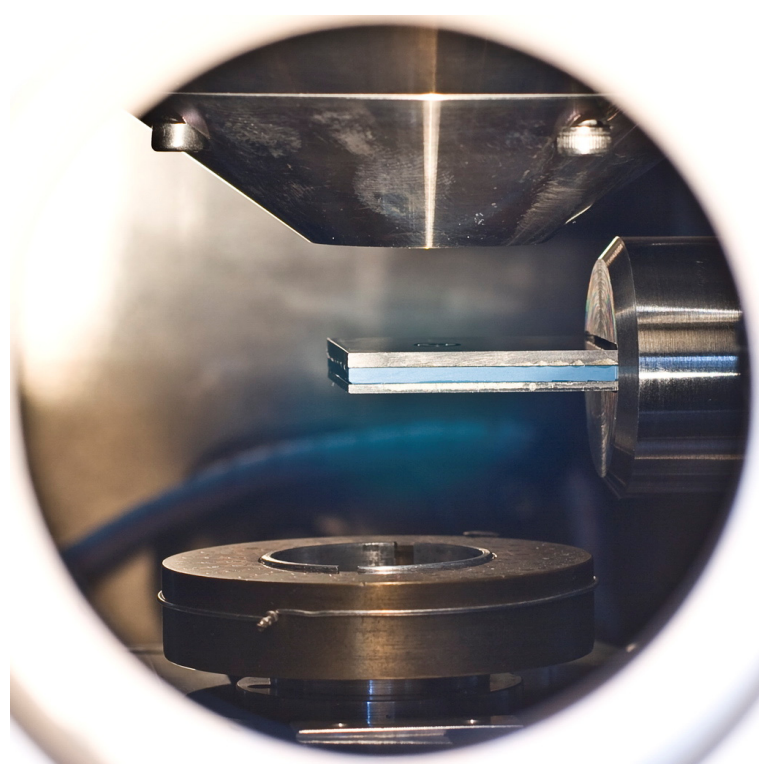

Fig. 3. Setup in a common SEM with pole piece (top) and sample (bottom).

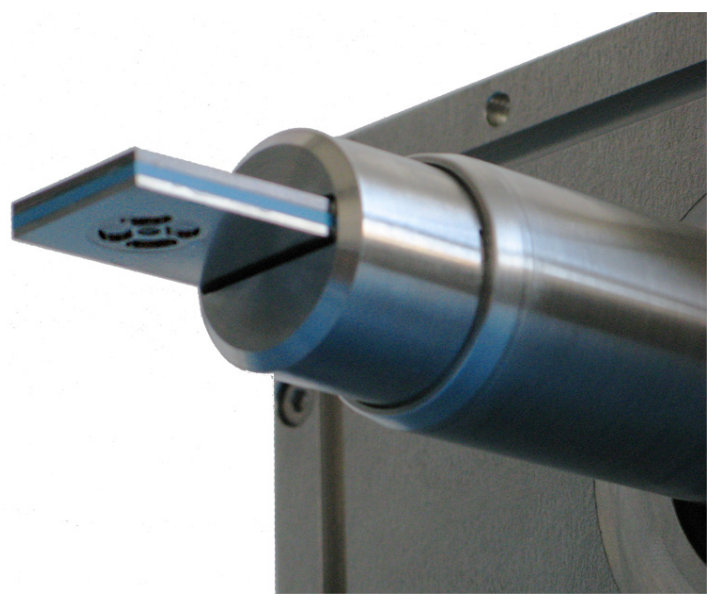

Fig. 2. Flat Quad detector (Quad XFlash 5060) which can be placed between the pole piece and the sample.

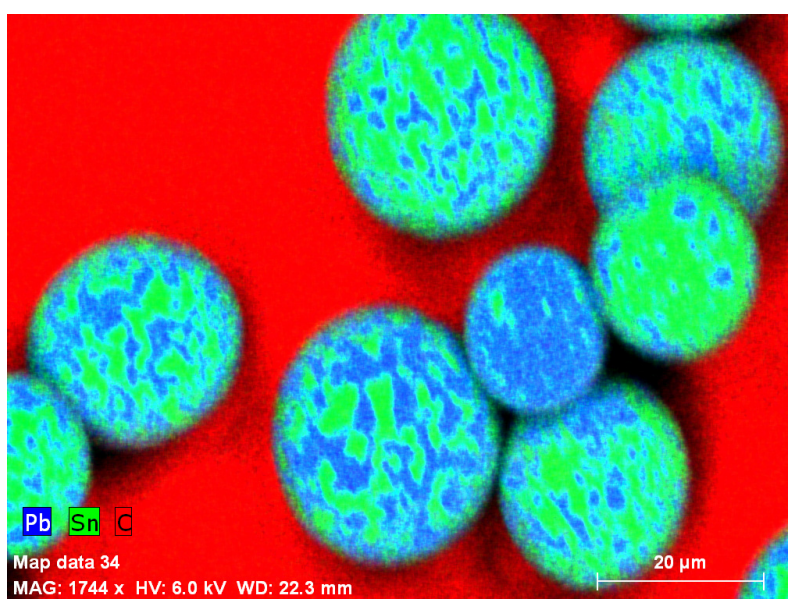

Fig. 4. A $1000 \times 750$ pixels spectral image containing a total of $46,000,000$ counts acquired within 30 minutes with a probe current of 100pA. 